\title{
Acute Visual Loss in Young Boy
}

\author{
Muhammad Saeed*, Memona Mohiuddin, Mohammad Hassan Al-Sumaili
}

Pediatric Medicine Department, King Fahad Armed Forces Hospital, KSA.

\begin{abstract}
Objective: Multiple sclerosis is immune mediated disorder of central nervous system (CNS). Diagnosis of multiple sclerosis is based on clinical findings and investigations, like MRI of CNS. Herein, we presents a 13-year old boy who presented with sudden loss of vision in both eyes for two days duration. The concurrent phenomenon of optic neuritis and multiple sclerosis is less common in children and rarely reported.
\end{abstract}

Keywords: Multiple sclerosis (MS), Optic neuritis (ON), Child, Steroids, Visual loss, Magnetic resonance imaging (MRI) brain, Fundoscopy.

\section{INTRODUCTION}

Multiple sclerosis (MS) in children, defined as state of clinical symptoms before the age of eighteen, although MS is a rare disorder in children. The overall incidence of MS in children ranges from 0.05 to 2.5 per 100,000 children [1]. There are different phenotypic differences in MS, which include clinical findings, Magnetic resonance imaging (MRI) and laboratory investigations, between the adult and children.

The current McDonald diagnostic criteria can be applied to pediatric age group, if the first clinical presentation is not associated with encephalopathy. MS is challenging diagnosis in pediatric age because of atypical presentation, MRI findings, and broad spectrum of potential differential diagnosis $[2,3]$. Neuro-opthalmological clinical findings of MS are frequently seen in children, including Neuromyelitis Optica (NO). Approximately $20 \%$ of the children with MS can present with visual loss due to ON which is an inflammatory condition of the optic nerve that results in loss of vision.

Herein, we report a boy with MS who presented with acute visual loss.

\section{CASE REPORT}

A 13-year old boy presented to our clinic with sudden onset of visual loss occurring over two days period. Few days ago he had noticed blurring of vision but he did not mention to his parents. It was also associated with pain on movement of the eyes. There was no any significant history of preceding illness or family history of autoimmune disorders. During admission his eye examination showed visual acuity of both eyes were just counting the fingers.

On examination, visual acuity was just perception of light, and on side counting fingers at $20 \mathrm{~cm}$ with minimally

*Address correspondence to this author at the Pediatric Medicine Department, King Fahad Armed Forces Hospital, KSA.

Email: muhammad964@hotmail.com preserved colour vision. Visual acuity on formal testing was $1 / 60$ bilaterally. There were fully range of conjugate movements of the eyes and pain free. A relative afferent papillary defect (RAPD) was noted in the left eye. Colour vision at presentation was left $0 / 17$ and right $1 / 17$. Fundoscopy examination revealed hyperemic and swollen optic disc bilaterally (Fig. 1). Visual field examination showed central scotoma. Rest of the CNS examination was unremarkable.

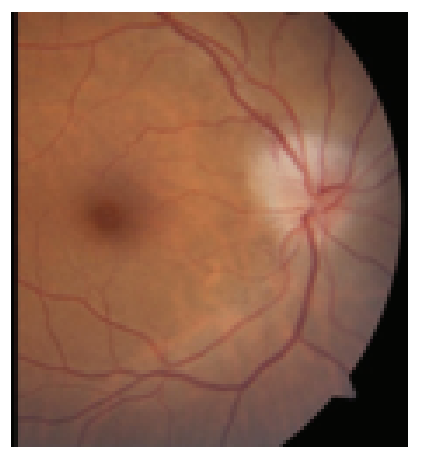

Fig. (1). Optic Neuritis, Fundus Appearance, Hyperemic and Swollen Optic Disc.

Diagnosis of bilateral ON was made based on ophthalmological examination. MRI of brain and optic nerve with gadolinium was performed which showed thickening of optic nerves bilaterally and typical discrete periventricular (Dawson's fingers) area of demyelination (Fig. 2). His blood examination for inflammatory markers were normal. Cerebrospinal fluid (CSF) analysis showed normal cell count but oligoclonal bands were positive. Vitamin D level was also normal. So based on clinical findings and investigations diagnosis of MS was made and child was treated with intravenous methylprednisolone one gram daily for five days and followed by oral steroids for four weeks. His vision improved after five days. At the time of discharge on 10th day his visual acuity was normal without any visual loss. 


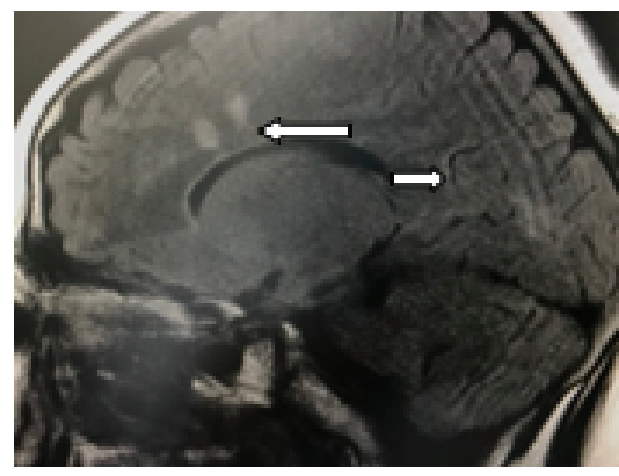

Fig. (2). Demylinating Lesions Suggestive of Multiple Sclerosis.

\section{DISCUSSION}

There is significant increase in the prevalence of MS in pediatric population of Saudi Arabia but still less than compare to western world and in the neighboring countries. Current estimated prevalence of MS is 7.7/100,000 population [4]. Common neurological manifestations include visual impairment, sensory disturbances, and difficulty in maintenance of balance, bladder dysfunction, cognitive problems, motor weakness and mood instability [5].

Optic neuritis in one of the commonest clinical manifestations that occur preceding MS. Approximately $15 \%$ to $20 \%$ of children with MS can present with optic neuritis [6].

Inflammation in MS of different parts of the brain like, transversemyelitis (TM), ON, brain stem dysfunction can be monophaisc that may be followed by further new episodes of neurological functions [7]. The possibility of multiphasic demyelinated encephalomyelitis (MDEM) also needs to be kept in consideration in pediatric age group with clinical features suggesting MS. Acute disseminated encephalomyelitis (ADEM) is a monophasic disease, which presents clinically with disturbed sensorium, convulsions and focal neurological deficit [8].

It is very important to differentiate MS from ADEM because it has prognostic importance. Children with ADEM have good prognosis as compare to MS which is thought to have a significant disability. Corticosterids is still choice of treatment for ON [9]. However, other treatment options include, immunoglobulins infusion and interferon B- $1 \alpha$ is one of the treatment for ON. Intravenous steroids are still the mainstay of treatment for $\mathrm{ON}$. The prognosis of $\mathrm{ON}$ in pediatric is relatively good. Bilateral $\mathrm{ON}$ is more common in children and $20 \%$ end up with visual disability. But our patient showed excellent response to steroids and improved over few days period.

In conclusion, MS is rare disease in children and it should alert the pediatrician about this disorder. Explanation should be given to the parents about the risks of progression of MS and its complications especially those present with ON.

\section{AUTHORS' CONTRIBUTION}

- Muhammad Saeed: Did writing, reviewing and collection of data.

- Memona Mohiuddin: Provided literature and helped in manuscript writing.

- Mohammad Hassan Al-Sumaili: Provided literature and helped in manuscript writing.

\section{CONFLICT OF INTEREST}

Declared none.

\section{ACKNOWLEDGEMENTS}

I would like to acknowledge Dr. Memona Mohiuddin and Dr. Mohammad Hassan Al-Sumaili for their efforts.

I would like to thank encouragement and enlightenment, provided from NJHS editorial board, Mr. Mohiuddin Chawla and Dr. Tahir Shamsi. Special thanks to the Editorial Manager Ms. Maria Baig for her prompt replying always and guidance.

\section{REFERENCES}

[1] Renoux C, Vukusic S, Mikaeloff Y, Edan G, Clanet M, Dubois B. Natural history of multiple sclerosis with childhood onset. N Engl J Med 2007; 356(25): 2603-13. DOI: 10.1056/NEJMoa067597

[2] Jeong A, Oeska DM, Holman J. Epidemiology of pediatric-onset multiple sclerosis: A systematic review of the literature. J Child Neurol 2019; 34(12): 705-12. DOI: $10.1177 / 0883073819845827$

[3] McDonald WI, Compston A, Edan G, et al. Recommended diagnostic criteria for multiple sclerosis: Guidelines from the international panel on the diagnosis of multiple sclerosis. Ann Neurol 2001; 50(1): 121-7. DOI: 10.1002/ana.1032

[4] Al-Jumah M, Bunyan R, Al Otaibi H, et al. Rising prevalence of multiple sclerosis in Saudi Arabia, a descriptive study. BMC Neurol 2020; 20(1): 49. DOI: 10.1186/s12883-020-1629-3

[5] Wallin MT, Culpepper WJ, Campbell J, et al. Global, regional, and national burden of multiple sclerosis 1990-2016: A systematic analysis for the global burden of disease study 2016. Lancet Neurol. 2019; (18): 269-85.

[6] Pérez-Cambrodí RJ, Gómez-Hurtado Cubillana A, Merino-Suárez ML, et al. Opticneuritis in pediatric population: A review in current tendencies of diagnosis and management. J Optom 2014; 7(3): 125-30. DOI: 10.1016/j.optom.2013.12.008

[7] Thompson AJ, Banwell BL, Barkhof F, et al. Diagnosis of multiple sclerosis: 2017 revisions of the McDonald criteria. 
Lancet Neurol 2017; $17 \quad$ (2): 162-73. $\quad$ DOI: 10.1016/S1474-4422(17)30470-2

[8] Dale RC, Branson JA. Acute disseminated encephalomyelitis or multiple sclerosis: can the initial presentation help in establishing a correct diagnosis? Arch Chil Dis 2005; 90(6): 636-9. DOI: 10.1136/adc.2004.062935
[9] Moss HE, Gao W, Balcer LJ, Joslin CE. Association of race/ethnicity with visual outcomes following acute optic neuritis: An analysis of the Optic Neuritis Treatment Trial. JAMA Ophthalmol 2014; 132(4): 421-7. DOI: 10.1001/jamaophthalmol.2013.7995

(C) 2021 National Journal of Health Sciences.

This is an open-access article. 